\title{
Children with borderline intellectual functioning and autism spectrum disorder: developmental trajectories from 4 to II years of age
}

This article was published in the following Dove Press journal:

Neuropsychiatric Disease and Treatment

4 October 2017

Number of times this article has been viewed

\author{
Martina Barnevik Olsson ${ }^{1,2}$ \\ Anette $\mathrm{Holm}^{3}$ \\ Joakim Westerlund ${ }^{1,4}$ \\ Åsa Lundholm Hedvall1,3 \\ Christopher Gillberg' \\ Elisabeth Fernell' \\ 'Gillberg Neuropsychiatry \\ Centre, Institute of Neuroscience \\ and Physiology, Sahlgrenska \\ Academy, Gothenburg University, \\ Gothenburg, ${ }^{2}$ PRIMA Child and \\ Adult Psychiatry, ${ }^{3}$ Department \\ of Psychology, Astrid Lindgren \\ Children's Hospital, ${ }^{4}$ Department of \\ Psychology, Stockholm University, \\ Stockholm, Sweden
}

Correspondence: Martina Barnevik

Olsson

PRIMA Barn och Vuxenpsykiatri,

Götgatan 7I S-II62I Stockholm, Sweden

Tel +4676 6479724

Email martina.barnevik@prima.se
Background: Studies on autism have tended to focus either on those with intellectual disability (ie, those with intellectual quotient [IQ] under 70) or on the group that is referred to as "highfunctioning", that is, those with borderline, average or above average IQ. The literature on cognition and daily functioning in autism spectrum disorder combined specifically with borderline intellectual functioning (IQ 70-84) is limited.

Methods: From a representative group of 208 preschool children diagnosed with autism spectrum disorder, those 50 children in the group with borderline intellectual functioning at ages 4.5-6.5 years were targeted for follow-up at a median age of 10 years. A new cognitive test was carried out in 30 children. Parents were interviewed with a semi-structured interview together with the Vineland Adaptive Behavior Scales $(n=41)$ and the Autism-Tics, attention-deficit/ hyperactivity disorder (AD/HD) and other comorbidities inventory (A-TAC) (n=36).

Results: Most children of interviewed parents presented problems within several developmental areas. According to A-TAC and the clinical interview, there were high rates of attention deficits and difficulties with regulating activity level and impulsivity. Vineland Adaptive Behavior Scales composite scores showed that at school age, a majority of the children had declined since the previous assessment at ages between 4.5 and 6.5 years. Almost half the tested group had shifted in their IQ level, to below 70 or above 84 .

Conclusion: None of the children assessed was without developmental/neuropsychiatric problems at school-age follow-up. The results support the need for comprehensive follow-up of educational, medical and developmental/neuropsychiatric needs, including a retesting of cognitive functions. There is also a need for continuing parent/family follow-up and support.

Keywords: AD/HD, A-TAC, autism spectrum disorder, borderline intellectual functioning, developmental disorders, Vineland

\section{Introduction}

Symptoms in children with autism spectrum disorder (ASD) change with age. Important factors predicting outcome include the child's intellectual level and presence of communicative speech at around age 5 years. ${ }^{1-4}$

Several studies support the conclusion that early detection/early intervention in children with ASD is related to better outcome. However, the interaction between intervention, autism symptoms and IQ changes has not been explained. ${ }^{5-7}$ Cognitive ability in autism is associated with autism severity, that is, children with intellectual disability (ID) have more severe autism symptoms than those with borderline intelligence or "normal" IQ. ${ }^{6}$ 
The literature on cognition and daily functioning in ASD combined with borderline intellectual functioning (BIF) (IQ 70-84) is very limited. Baglio et $\mathrm{al}^{8}$ described the group with BIF as a "marginal clinical category". They emphasized that children with BIF typically show a heterogeneous neuropsychological profile with impact on the quality of everyday life. ${ }^{8}$

In several previously published studies, we have followed a group of preschool children with ASD over a period of 2 years with regard to change and stability of intellectual level, ASD severity and adaptive functioning. ${ }^{9-11}$ Subgroups have been followed over a period of 7 years. ${ }^{12,13}$ Diagnoses of ID and autism/autistic disorder have been found to be relatively stable, whereas BIF and pervasive developmental disorder-not otherwise specified/atypical autism have been more variable over the 2-year period. ${ }^{11}$ At 2-year follow-up, 50 of the 198 children (25\%) with ASD, tested with regard to their general intellectual functioning, were found to have BIF. Children with the combination of ASD and BIF have often been referred to as suffering from high-functioning autism. ${ }^{1,2}$ Another $25 \%$ had average intellectual functioning (AIF), and $\sim 50 \%$ had ID.

We wanted to examine the ASD group with BIF again, several years later in order to find out what had happened to them, both in terms of developmental/psychiatric/adaptive functioning and as regards further intervention needed. The aims of this study were, therefore, to perform a new follow-up when the children were at young school age, to assess their current intellectual, adaptive, developmental, behavioral and ASD profiles. A particular objective was to analyze the proportions that might have changed their intellectual functioning status from BIF to ID or to AIF.

\section{Methods}

\section{Participants}

\section{Original study group}

The original study group consisted of the 313 preschool children (birth-years 2002-2006) in the county of Stockholm, who had been diagnosed with ASD before 4.5 years of age. Of these 313 children, 25 had been referred to their local habilitation center, mainly due to complex medical needs and severe multi-impairments, for example, those whose ASD was due to a severe genetic syndrome. In this group, autism was not considered to be the child's major impairment, instead other problems dominated the clinical picture. The remaining 288 children had been referred to the Autism Centre for Young Children (ACYC) in Stockholm for intervention. However, 24 had been referred to the center prior to start of the study, 37 families declined participation,
15 families could not communicate in either Swedish or English, 2 children had moved abroad, and another 2 had been referred to local habilitation centers due to complex medical needs. Thus, the original study group comprised 208 children (176 boys and 32 girls). ${ }^{9}$ The children had been thoroughly psychiatrically, and physically examined at the time of inclusion in the study (Time 1) before interventions started, and 198 were also examined in depth at follow-up 2 years later (Time 2). Also in this group, many different medical conditions were represented. ${ }^{14}$ Interventions had been of different intensities and all based on applied behavior analysis (ABA), intensive ( $>25$ hours per week) or non-intensive ( $<15$ hours per week). ${ }^{10}$ Of the 198 children, 50 had BIF, 51 had AIF, 95 had ID and 2 children had not had an IQ test at the Time 2 (T2) assessment ${ }^{10}$ (Figure 1).

\section{Time 3 (T3) study group}

The present study group consisted of 50 children with BIF; 46 boys and 4 girls, age range $9-13$ years (median 10 years). At initial referral to the ACYC, all children had a diagnosis of ASD. At the 2-year follow-up (T2), at ages 4.5-6.5 years, they were found to have, in addition to ASD, various other developmental deviations in areas of speech and language, attention and activity regulation and behavioral problems.

\section{Procedure}

At T3, the parents of the children were once again contacted by letter and telephone and different interviews - one clinical semi-structured interview and a Vineland interview Sparrow et $\mathrm{a}^{15}$ - were performed by the first author (MBO) and another interview, the Autism-Tics, attention-deficit/hyperactivity disorder (AD/HD) and other comorbidities inventory $(\mathrm{A}-\mathrm{TAC})^{16-19}$ over the telephone, by an experienced layperson from a market research center. In addition, a cognitive test performed by a psychologist (AH) was included at T3.

\section{Measures}

\section{Parental semi-structured interview}

A semi-structured telephone interview with a qualitative approach was performed with one of each child's parents, to obtain information about the child's current situation both in school and at home. Questions on type of school and school support were raised, as were questions pertaining to different developmental domains; speech and language, social abilities, activity and impulsivity regulation, attention span and externalizing behavior. Parents were asked if the family had any ongoing contacts with a pediatrician/neuropediatrician, child psychiatrist or habilitation center and if the parents found that the services they received met their needs. 


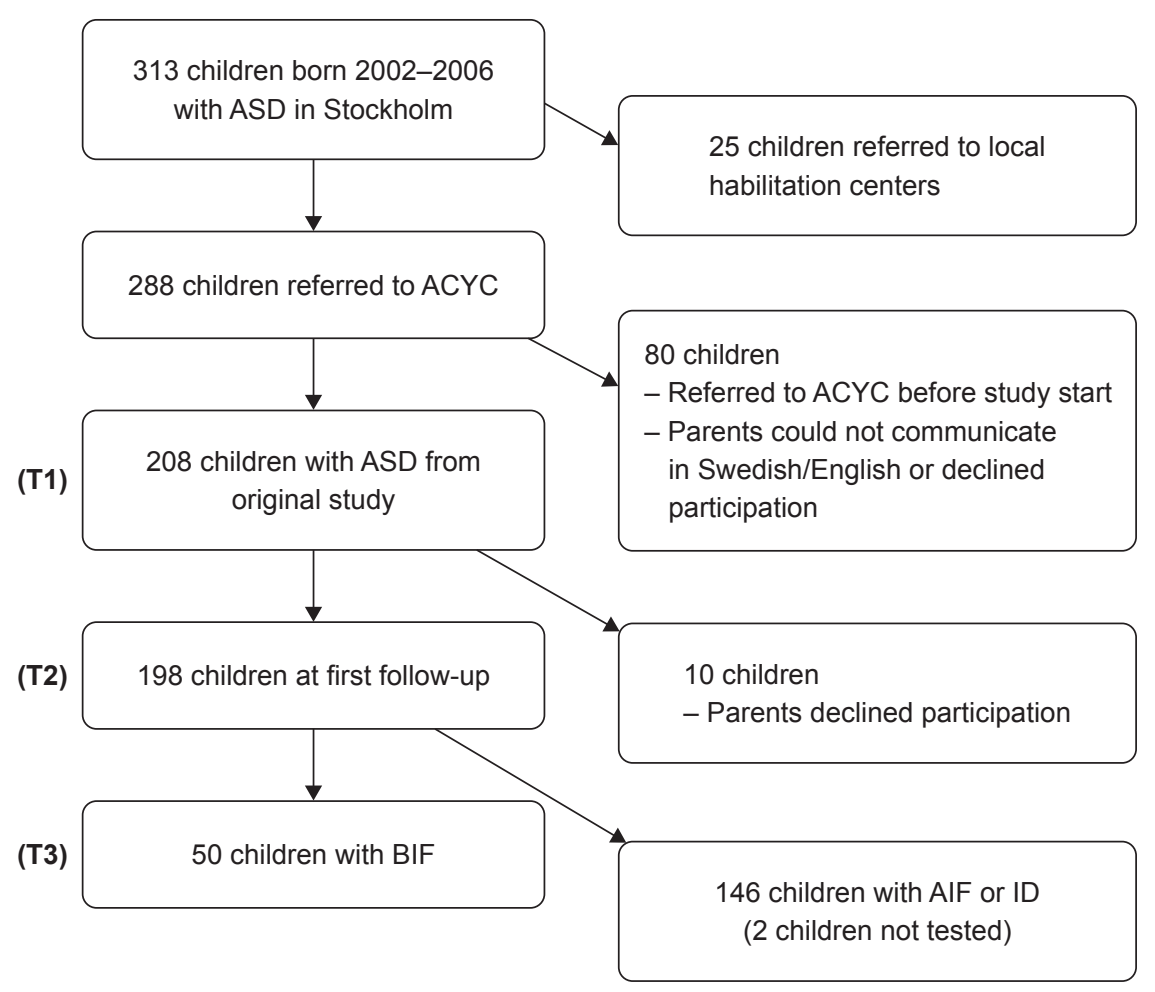

Figure I Flowchart demonstrating the inclusion procedure and study group.

Note: $\mathrm{TI}, \mathrm{T} 2$, and $\mathrm{T} 3=$ Time I, Time 2, and Time 3 respectively.

Abbreviations: ACYC, Autism Centre for Young Children; AIF, average intellectual functioning; ASD, autism spectrum disorder; BIF, borderline intellectual functioning; ID, intellectual disability.

\section{The autism - tics, AD/HD and other comorbidities inventory (A-TAC) interview}

The A-TAC ${ }^{16-19}$ interview was performed with one of the children's parents. The A-TAC is a screening interview focusing on virtually all common child and adolescent psychiatry and is designed to be used by laymen over the phone.

The A-TAC has been validated against clinical diagnoses cross-sectionally ${ }^{16,17}$ and longitudinally ${ }^{19}$ and has been found to be a sensitive tool to screen for $\mathrm{ASD}, \mathrm{AD} / \mathrm{HD}$, tics, learning disorder and developmental coordination disorder (DCD). ${ }^{17}$ For ASD, AD/HD and learning disorder, 2 cutoff-levels exist 1) "high", which is a proxy for the clinical diagnosis with moderate sensitivity but high specificity, and 2) "low", which is a broad screening with high sensitivity but moderate specificity designed to capture pronounced subthreshold traits. ${ }^{17,19}$

\section{Vineland adaptive behavior scales (VABS) interview}

An interview was also performed at T3 according to the VABS, ${ }^{15}$ with one of the child's parents. This interview included communication, daily living skills and social domains, as well as a composite score. The interview was administered to a parent or caregiver using a semi-structured format, which provided a targeted assessment of adaptive behavior. The interview was given over the telephone, taking 45-60 minutes. A subgroup of all the 50 children had complete VABS-II results, both at first (Time 1, T1) and second $\mathrm{T} 2$ assessment.

\section{Cognitive testing}

Parents of those 50 children who at T2 had ASD and BIF, were offered a new cognitive test of their child (Wechsler Intelligence Scale for Children-fourth edition [WISC-IV] ${ }^{20}$ ). At T2, all had been tested with the Wechsler Preschool and Primary Scale of Intelligence-third edition (WPPSI-III). ${ }^{21}$ WISC-IV includes verbal comprehension index, perceptual reasoning index, working memory index and processing speed index, and gives a full scale IQ (FSIQ). The WISC-IV test was performed by one of the 2 earlier project psychologists (AH) and took place at Karolinska University Hospital in Stockholm. Test duration was about half a day per child. FSIQ at T3 was compared with FSIQ at T2 (all had had IQ 70-84 at that time). No comparisons with regard to the different IQ indices or subtests of the WISC-IV were made. 


\section{Statistical analyses}

Differences between the 3 time points with regard to mean Vineland composite scores were analyzed with a repeated measures analysis of variances (ANOVA). In this analysis, partial etasquared $\left(\eta_{\text {partial }}^{2}\right)$ was used as a measure of effect size. The ANOVA was followed up by post hoc analysis (Bonferroni) in order to study differences between specific time points. An alpha level of 0.05 was used for all statistical analyses.

\section{Ethical approval}

All parents provided verbal informed consent to participate in the study. The study was approved by the Ethical committee at Karolinska Institute, 2012-05-15 2012/734-32.

\section{Results}

Of the 50 children, parents of 49 (45 boys and 4 girls, age range $9-13$ years, mean age 10.4 years) could be reached. For one family, contact details could not be obtained. Six families declined participation in a new follow-up (had moved away, family situation, lack of time). Parents of 43 children accepted to take part in all or parts of the follow-up. Parents of 41 children participated in the semi-structured interview and in the Vineland interview, parents of 36 children participated in the A-TAC interview, and 30 children had a cognitive test according to WISC-IV, of which 2 assessments had been carried out at other clinics, but copies of the tests were obtained.

\section{Semi-structured interview}

\section{School situation}

Of the 41 interviewed parents, all but 3 children studied the normal curriculum. These 3 studied according to a specialized curriculum for children with ID. Twenty-five children went to regular schools. Ten of these had a special needs assistant of their own, while 6 children shared an assistant with classmates. Nine children had very little individual support. Thirteen children studied at specialized schools for children with autism. Ten of the 41 parents clearly expressed that their children did not have enough support in school.

Parents especially pointed to the children's need of more individual support at school; that is, help with structure and planning, and with initiating and completing school assignments. Another aspect, related to the children's executive dysfunctions, that was emphasized by parents was the need for better cooperation between the school and home with regard to what homework the child was expected to accomplish.

\section{AD/HD-symptoms}

Out of the 41 interviewed parents, 34 reported that their child had problems regarding attention and 21 of these children also had hyperactivity and/or impulsivity. The reported symptoms within the $\mathrm{AD} / \mathrm{HD}$ spectrum were mainly in accordance with the situation at T2 and 16 of the 41 children (39\%) had now received a diagnosis of $\mathrm{AD} / \mathrm{HD}$ at a clinical assessment after T2. At T3, a total of 13 of the $41(32 \%)$ children had pharmacological treatment for $\mathrm{AD} / \mathrm{HD}$.

\section{Speech and language}

A large number, 31 of the 41 interviewed parents, reported that their child had problems with speech and language (problems with at least one of the following language domains; grammar, expressive speech, vocabulary, pragmatic deficiency and articulation). Of these children, 10 had or had had a regular contact with a speech pathologist and/or attended or had attended a special speech/language class.

\section{Behavior}

Parents of 34 of the 41 children reported that their child had behavioral problems interfering with many daily life situations. In 7 of these children, the problems were severe (externalizing and often aggressive). Four of the 7 children also had clear indications of attention/hyperactivity/impulsivity symptoms. Eleven children had a very inflexible behavior. Parents of 3 children in the total group reported obsessivecompulsive problems in their children. Four children had severe anxiety and 5 had verbal and/or motor tics.

\section{Regular contacts with pediatric/ psychiatric or habilitation services}

All 50 children were "entitled" to contact with habilitation services but of the 41 interviewed parents, only 12 reported that their child had such a contact. Fourteen of 41 children had some contact with the Child and Adolescent Mental Health Service (CAMHS) and 10 children met with a pediatrician/ neuropediatrician on a regular basis. Two of these had a diagnosis of epilepsy. Seventeen children (about 40\%) had no regular contact with a physician.

\section{The autism - tics, AD/HD and other comorbidities inventory (A-TAC) interview}

Most children of the 36 interviewed parents presented problems within several areas. Five modules were targeted; $\mathrm{ASD}, \mathrm{AD} / \mathrm{HD}$, tic disorder, $\mathrm{DCD}$ and learning disorder. Of the 36 children, 21 had symptom levels corresponding to 


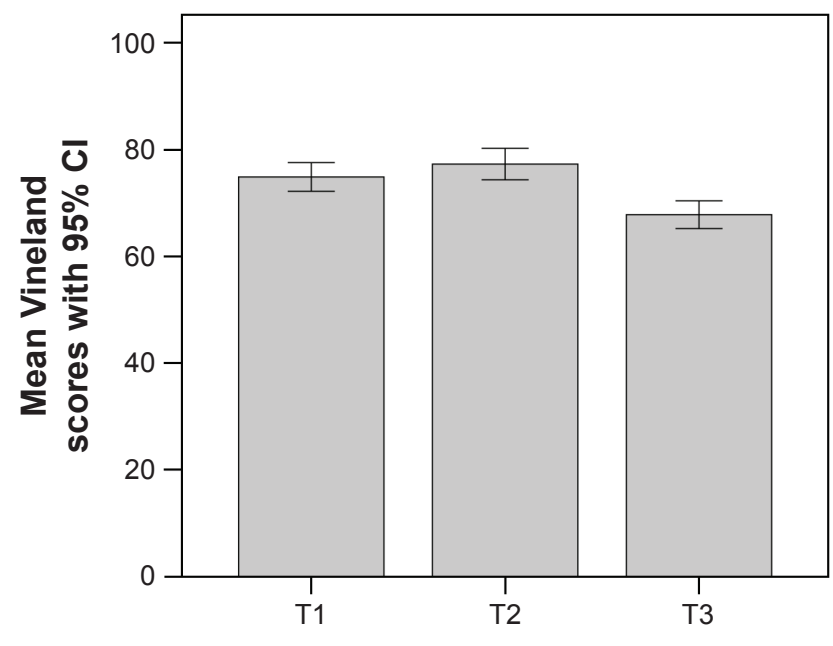

Figure 2 Mean Vineland scores with $95 \% \mathrm{Cl}$ for the 3 assessment times. Note: TI, T2, and T3 = Time I, Time 2, and Time 3 respectively.

a clinical proxy of ASD and another 11 reached the cutoff for the broad screening diagnosis. Ten children had symptom levels corresponding to a clinical proxy of $\mathrm{AD} / \mathrm{HD}$, and another 15 encompassed the broad screening cut-off. 13 children had symptom levels corresponding to tic disorder and 6 had DCD (clinical proxy). Five had symptom levels corresponding to learning disorder (clinical proxy), while 18 reached cut-off for the broad screening diagnosis.

\section{Vineland adaptive behavior scales}

A repeated measures ANOVA with Time (1-3) as the withinsubject factor and rating on the VABS as the dependent variable showed a significant effect of Time $(F 2,80=28.24$, $p<0.001, \eta^{2}=0.414$ ), see Figure 1. Post-hoc tests (Bonferroni) showed that the children had got a significantly lower mean rating at T3 as compared with both T1 $(p<0.001)$ and T2 $(p<0.001)$. The difference between T1 and T2 was, however, not significant $(p=0.155)$ (Figure 2).

\section{Cognitive testing}

Of the 50 children, 30 had a cognitive test according to WISC-IV (of which 2 were outside the study but copies of tests were obtained). In total, 6 children now had an FSIQ clearly below 70, 7 had FSIQ of 85 or above and 17 children still had results between 70 and 84, that is, in the BIF area. All 6 children with FSIQ below 70, had Vineland composite scores supporting ID.

\section{Discussion}

The present research set out to study the cognitive and adaptive outcomes at mid-school age in a group of 50 children diagnosed at preschool age with a combination of ASD and below average IQ, that is, BIF. These children were part of a larger cohort of about 200 children with ASD at IQ levels ranging from severe ID to above average functioning - the majority of whom had recently been followed up..$^{13}$ The present study focused on the group that, based on results from previous studies, we hypothesized might have the least stable/predictable outcomes. ${ }^{14,22}$

Only about $60 \%$ of the target group participated in all the follow-up assessments, but about $85 \%$ were evaluated on at least one of the measures included. The major finding was that the majority of the children had several developmental/ neuropsychiatric problems at this school-age follow-up.

With regard to the Swedish school system, children with ID are entitled to study according to a curriculum adapted for pupils with learning impairments. Children with other cognitive disorders, such as autism, study according to the regular curriculum. The current curriculum for compulsory schools promotes all pupils to be educated in general classes and there is a need to specify when segregated options are considered for some pupils. In our study group, only onethird of the children with ASD and BIF had their education in special classes for children with autism, although parents' reports indicated that more children in the study group would benefit from that type of school placement.

According to results from A-TAC and the clinical interview, there were high rates of children with attention deficits and difficulties with regulating activity level and impulsivity. With regard to ASD, around $90 \%$ of the children still met criteria for ASD or had definite subthreshold symptoms; two-thirds of these had symptoms corresponding to clear DSM-ASD. Almost 30\% had symptoms corresponding to ADHD and around $40 \%$ had symptoms of subthreshold severity. More than $30 \%$ of the children with $\mathrm{AD} / \mathrm{HD}$ had pharmacological treatment. Of the 32 children at or above high or low cut-off scores for ASD in the A-TAC interview, 23 also had at or above high or low cut-off scores for $\mathrm{AD} / \mathrm{HD}$, thus supporting the high co-occurrence between the 2 disorders. There are many studies reporting strong relationships between ASD or social-communication traits, and $\mathrm{AD} / \mathrm{HD}$ or hyperactive traits in children and adolescents, supporting the evidence for the co-existence of ASD and AD/HD. ${ }^{23}$

The semi-structured interview revealed that 3 out of 4 children had problems with speech and language. Behavioral problems were common and were reported in $>80 \%$ of the children, one in 5 of whom had severe, externalizing problems. In a study of tantrum profiles in children, aged 3-16 years, with ASD, AD/HD and combined ASD and $\mathrm{AD} / \mathrm{HD}$, it was found that tantrum behaviors were observed 
at relatively high levels in children with ASD alone, AD/HD alone and in children with comorbid ASD and AD/HD, and that tantrum behavior was highest in the combined ASD + AD/HD group. ${ }^{24}$

No child was without developmental/neuropsychiatric problems at this school-age follow-up, although many had subthreshold symptoms within areas of ASD and ADHD. The importance of also considering developmental/psychiatric symptoms and problems even if the full diagnostic criteria are not met, has been highlighted by other authors. In an adult functioning outcome study, risks were not limited to those who received a diagnosis; participants with subthreshold psychiatric problems also had higher odds of adverse adult outcomes. $^{25}$

VABS composite scores showed that a majority of the children at this time had declined since the former period of measurement. One can only speculate as to the reasons for this negative trend. In our original study group comprising 200 children, of whom about one-fourth constituted the group with ASD and BIF, all had received different types of intervention based on $\mathrm{ABA}$ during the 2-year period at the ACYC. ${ }^{10}$ It is likely that both the children and their parents were helped by the earlier ABA interventions, which in some children had been of intensive type and in some of a targeted type (usually delivered by the ACYC), by parental education and general family support from the ACYC. The behavioral intervention was terminated after 2 years, and parent training and general family support were also reduced as the children grew older. At the same time, higher demands were being placed on the children in school compared with preschool, which all may have contributed to the declining trend. On top of this, many do not have sufficient support in school according to their needs and as many as one in four parents clearly expressed that their child had insufficient support at school.

Our result accords with the study by Kanne et al, ${ }^{26}$ investigating adaptive behavior profiles in children and adolescents with ASD, mean age 9 years, with varying IQ sores. They found that Vineland Composite was negatively associated with age; older individuals have a greater gap between IQ and adaptive skills and that the gap between IQ and adaptive impairments decreased in lower functioning individuals with ASD, while adaptive behavior scores in the high IQ group were below the standard IQ score. They concluded that IQ was a strong predictor of adaptive behavior.

In a study by Szatmari et al, ${ }^{27}$ developmental trajectories in children with ASD were found to be heterogeneous and 3 distinct trajectory groups; worsening, stable and improving, with regard to adaptive functioning were identified. It was emphasized that this heterogeneity should necessitate a flexible suite of interventions, targeting each child's strengths and difficulties.

The cognitive test supported the hypothesis that there is considerable instability of BIF at a group level in children with ASD tested at young ages. Of the 30 children tested, $20 \%$ now had an FSIQ of below 70, corresponding to ID, another $23 \%$ now had an average IQ, at or above 85 , and a little more than half the group had stayed within the original IQ-band of BIF. We cannot claim that these changes are significant, different test methods had been used at T2 and T3. However, the tools are designed to measure the same construct ${ }^{28}$ and results illustrate the varying cognitive/ developmental trajectories in children with BIF. Our cognitive results, although not statistically substantiated, support the instability of an IQ in the BIF area ${ }^{11}$ and the need of follow-up assessments of cognitive functions. Children with BIF and ASD should be legislatively re-tested so that their educational needs can be appropriately met.

The majority of children in our study group had a combination of ongoing problems that accorded with the umbrella term of ESSENCE (Early Symptomatic Syndromes Eliciting Neurodevelopmental Clinical Examinations), ${ }^{29}$ in spite of the fact that they had received appropriate intervention for 2 years in early childhood. The vast majority of children in this group, besides ASD and AD/HD, had other behavioral problems and language difficulties. Our current societal system to a large extent restricts services to certain diagnoses, such as ID \pm ASD. This means that many children with combinations of developmental problems; autistic symptoms, hyperactivity/impulsivity, attention, language and behavioral problems, BIF - but not formal ID - do not receive appropriate support from habilitation or other specific societal support systems.

Children with ASD combined with BIF are often referred to as high-functioning children, although their cognitive level is not within the high but rather within the low "normal" variation. The term high-functioning may therefore in this group be somewhat misleading. Our results highlight the need for repeated cognitive assessments in all children with ASD in order to provide best possible education and support. All children in our study group were, due to their ASD, "entitled" to contact with habilitation services under Swedish law, but only about one third of the children had such contact. About half the group had contact with the CAMHS or saw a pediatrician on a regular basis, but several families had been left to their own devices. 
Limitations of the study include that only 30 of the 50 children with BIF at the T2 follow-up could be assessed with a new cognitive test at $\mathrm{T} 3$ and that differences were only reported with regard to FSIQ without any statistical measures. Strengths included that the children belonged to a community based, representative group, $>80 \%$ had parents who took part in both the Vineland and the semi-structured interview (which corresponded well to one another) and that the A-TAC interview is well validated against clinically ascribed diagnoses. Another strength would be the focus on the BIF of below average IQ group, as few studies have specifically highlighted these children in follow-up studies.

In conclusion, our study revealed that children diagnosed at preschool age with ASD combined with BIF, after appropriate intervention for 2 years, still need to be followed up for several more years. The child's ASD was generally combined with other developmental disorders or problems, that is, the children had "autism plus". ${ }^{30}$ Carrying out follow-up assessments continuously throughout the school years will yield a more valid estimation of the children's developmental status and a better basis for realistic educational planning and intervention. This group is in continued need of support; educationally, medically and with regard to family support.

\section{Acknowledgments}

Financial support was given through a grant support from Prima Child and Adult Psychiatry (MBO), from Kempe Carlgrens Foundation and from Per and Annmari Ahlqvist Foundation (CG). We are much obliged to Isabella Kizling, Karolinska Institute and Sebastian Lundström, GNC for help with interpretation of A-TAC, Eva Billstedt, GNC, for help with cognitive data and to the families who have taken part in the study.

\section{Disclosure}

The authors report no conflicts of interests in this work.

\section{References}

1. Venter A, Lord C, Schopler E. A follow-up study of high-functioning autistic children. J Child Psychol Psychiatry. 1992;33(3):489-507.

2. Szatmari P, Bartolucci G, Bremner R, Bond S, Rich S. A follow-up study of high-functioning autistic children. J Autism Dev Disord. 1989; 19(2):213-225.

3. Szatmari P, Bryson SE, Boyle MH, Streiner DL, Duku E. Predictors of outcome among high functioning children with autism and Asperger syndrome. J Child Psychol Psychiatry. 2003;44(4):520-528.

4. Billstedt E, Gillberg IC, Gillberg C. Autism in adults: symptom patterns and early childhood predictors. Use of the DISCO in a community sample followed from childhood. J Child Psychol Psychiatry. 2007; 48(11):1102-1110.

5. Volkmar FR, Pauls D. Autism. Lancet. 2003;362(9390):1133-1141.
6. Ben Itzchak E, Lahat E, Burgin R, Zachor AD. Cognitive, behavior and intervention outcome in young children with autism. Res Dev Disabil. 2008;29(5):447-458.

7. Begovac I, Begovac B, Majić G, Vidović V. Longitudinal studies of IQ stability in children with childhood autism - literature survey. Psychiatr Danub. 2009;21(3):310-319.

8. Baglio G, Blasi V, Sangiuliano Intra F, et al. Social competence in children with borderline intellectual functioning: delayed development of theory of mind across all complexity levels. Front Psychol. 2016;7:1604.

9. Fernell E, Hedvall A, Norrelgen F, et al. Developmental profiles in preschool children with autism spectrum disorders referred for intervention. Res Dev Disabil. 2010;31(3):790-799.

10. Fernell E, Hedvall Å, Westerlund J, et al. Early intervention in 208 Swedish preschoolers with autism spectrum disorder. A prospective naturalistic study. Res Dev Disabil. 2011;32(6):2092-2101.

11. Hedvall Å, Westerlund J, Fernell E, Holm A, Gillberg C, Billstedt E. Autism and developmental profiles in preschoolers: stability and change over time. Acta Paediatr. 2014;103(2):174-181.

12. Olsson MB, Westerlund J, Lundström S, Giacobini M, Fernell E, Gillberg C. "Recovery" from the diagnosis of autism - and then? Neuropsychiatr Dis Treat. 2015;11:999-1005.

13. Barnevik Olsson M, Lundström S, Westerlund J, Giacobini MB, Gillberg C, Fernell E. Preschool to School in Autism: neuropsychiatric problems 8 years after diagnosis at 3 years of age. J Autism Dev Disord. 2016;46(8):2749-2755.

14. Eriksson MA, Westerlund J, Hedvall Å, Åmark P, Gillberg C, Fernell E. Medical conditions affect the outcome of early intervention in preschool children with autism spectrum disorders. Eur Child Adolesc Psychiatry. 2013;22(1):23-33.

15. Sparrow SS, Cicchetti DV, Balla DA. Vineland Adaptive Behavior Scales, Second Edition (Vineland $\left.{ }^{\mathrm{TM}}-\mathrm{II}\right)$. Circle Pines, MN: American Guidance Service; 2005.

16. Hansson SL, Svanström Röjvall A, Råstam M, Gillberg C, Anckarsäter H. Psychiatric telephone interview with parents for screening of childhood autism-tics, attention-deficit hyperactivity disorder and other comorbidities (A-TAC): preliminary reliability and validity. Br J Psychiatry. 2005;187:262-267.

17. Larson T, Anckarsäter H, Gillberg C, et al. The autism - tics, AD/HD and other comorbidities inventory (A-TAC): further validation of a telephone interview for epidemiological research. BMC Psychiatry. 2010;10:1.

18. Halleröd SL, Larson T, Ståhlberg O, et al. The Autism - Tics, AD/HD and other Comorbidities (A-TAC) telephone interview: convergence with the Child Behavior Checklist (CBCL). Nord J Psychiatry. 2010; 64(3):218-224.

19. Larson T, Lundström S, Nilsson T, et al. Predictive properties of the A-TAC inventory when screening for childhood-onset neurodevelopmental problems in a population-based sample. BMC Psychiatry. 2013; 13:233.

20. Wechsler D. WISV-IV. Wechsler Intelligence Scale for Children. Fourth Edition [Swedish version]. Stockholm: Psykologiförlaget AB; 2007.

21. Wechsler D. WPPSI-III. Wechsler Preschool and Primary Scale of Intelligence. [Swedish version]. Stockholm: Psykologiförlaget AB; 2005.

22. Baghdadli A, Assouline B, Sonié S, et al. Developmental trajectories of adaptive behaviors from early childhood to adolescence in a cohort of 152 children with autism spectrum disorders. J Autism Dev Disord. 2012;42(7):1314-1325.

23. Musser ED, Hawkey E, Kachan-Liu SS, et al. Shared familial transmission of autism spectrum and attention-deficit/hyperactivity disorders. J Child Psychol Psychiaty. 2014;55(7):819-827.

24. Goldin RL, Matson JL, Tureck K, Cervantes PE, Jang J. A comparison of tantrum behavior profiles in children with ASD, ADHD and comorbid ASD and ADHD. Res Dev Disabil. 2013;34(9):2669-2675.

25. Copeland WE, Wolke D, Shanahan L, Costello EJ. Adult functional outcomes of common childhood psychiatric problems: a prospective, longitudinal study. JAMA Psychiatry. 2015;72(9):892-899. 
26. Kanne SM, Gerber AJ, Quirmbach LM, Sparrow SS, Cicchetti DV, Saulnier CA. The role of adaptive behavior in autism spectrum disorders: implications for functional outcome. J Autism Dev Disord. 2011; 41(8):1007-1018.

27. Szatmari P, Georgiades S, Duku E, et al. Pathways in ASD Study Team. Developmental trajectories of symptom severity and adaptive functioning in an inception cohort of preschool children with autism spectrum disorder. JAMA Psychiatry. 2015;72(3):276-283.
28. Clark ML, Barbaro J, Dissanayake C. Continuity and change in cognition and autism severity from toddlerhood to school age. J Autism Dev Disord. 2017;47(2):328-339.

29. Gillberg C. The ESSENCE in child psychiatry: early symptomatic syndromes eliciting neurodevelopmental clinical examinations. Res Dev Disabil. 2010;31(6):1543-1551.

30. Gillberg C, Fernell E. Autism plus versus autism pure. J Autism Dev Disord. 2014;44:3274-3276.

\section{Publish your work in this journal}

Neuropsychiatric Disease and Treatment is an international, peerreviewed journal of clinical therapeutics and pharmacology focusing on concise rapid reporting of clinical or pre-clinical studies on a range of neuropsychiatric and neurological disorders. This journal is indexed on PubMed Central, the 'PsycINFO' database and CAS, and is the official journal of The International Neuropsychiatric Association (INA). The manuscript management system is completely online and includes a very quick and fair peer-review system, which is all easy to use. Visit http://www.dovepress.com/testimonials.php to read real quotes from published authors.

Submit your manuscript here: http://www.dovepress.com/neuropsychiatric-disease-and-treatment-journal 